\title{
Problemas emocionais e de comportamento e rendimento escolar em adolescentes
}

\author{
Bruna Mainardi Rosso Borba ${ }^{1}$ \\ Angela Helena Marin ${ }^{1}$ \\ ${ }^{1}$ Universidade do Vale do Rio dos Sinos, RS, Brasil
}

\begin{abstract}
Resumo
Trata-se de um estudo transversal do tipo correlacional e comparativo, com abordagem quantitativa, no qual avaliou-se a relação entre os problemas emocionais e de comportamento e o rendimento escolar de adolescentes a partir da avaliação destes, seus familiares e professores. Participaram 70 adolescentes e, em igual número, seus principais cuidadores, além de 21 professores. Os adolescentes responderam ao Inventário de Comportamentos Autorreferidos para jovens (YSR), as famílias ao Inventário dos Comportamentos de Crianças e Adolescentes (CBCL) e os professores, ao Inventário dos Comportamentos de Crianças e Adolescentes (TRF). O rendimento escolar foi avaliado a partir das notas obtidas nas disciplinas de português e matemática. Os resultados evidenciaram que os problemas de atenção, assim como os sintomas externalizantes foram os que mais se associaram ao baixo rendimento escolar. Ressalta-se, portanto, a importância de considerar os problemas emocionais e de comportamento no contexto escolar, a partir da perspectiva de múltiplos informantes, tendo em vista sua associação com o rendimento acadêmico de adolescentes.
\end{abstract}

Palavras-chave: Saúde mental; Distúrbios do comportamento; Rendimento escolar; Adolescência.

\section{Emotional and behavioral problems and academic achievement in adolescents}

\begin{abstract}
This is a transversal correlational and comparative study, with a quantitative approach, which evaluated the relation between emotional and behavioral problems and school performance of adolescents considering their evaluation, of their relatives and teachers'. Participated 70 adolescents, as well as their main caregivers, an equal number, and 21 teachers. The adolescents answered to Youth Self Report (YSR), the families to Child Behavior Checklist (CBCL), and the teachers to Teacher's Report Form (TRF). The school performance was evaluated from the grades of Portuguese and Math subjects. The results showed that attention problems, as well as externalizing symptoms were the scales most associated with low school performance. Therefore, it is important to consider the emotional and behavioral problems in the school context, from the perspective of multiple informants, in view of their association with adolescent academic performance.
\end{abstract}

Keywords: Mental health; Behavior disorders; Academic achievement; Adolescence.

\section{Problemas emocionales y de comportamiento y rendimiento escolar en los adolescentes}

\section{Resumen}

Se trata de un estudio transversal de correlacional y comparativo, con un enfoque cuantitativo, en el que se evaluó la relación entre los problemas emocionales y de comportamiento y el rendimiento escolar de los adolescentes a partir de la evaluación de éstos, sus familias y profesores. Participaron 70 adolescentes y sus principales cuidadores en igual número, y 21 profesores. Los adolescentes respondieron al inventario de conductas de auto-reporte de la juventud (YSR), las familias del Inventario de los maestros del niño y el comportamiento de los adolescentes (CBCL) y el Inventario de Niños y Adolescentes Comportamiento (TRF). El rendimiento escolar se evaluó sobre la base de las calificaciones obtenidas en las disciplinas de portugués y matemática. Los resultados mostraron que los problemas de atención, así como los síntomas exteriorizados se asocian con más bajo rendimiento escolar. Se resalta, por lo tanto, la importancia de considerar los problemas emocionales y de comportamiento en el contexto escolar, desde la perspectiva de múltiples informantes, teniendo en vista su asociación con el rendimiento académico de adolescentes.

Palabras clave: Salud mental; Trastornos de la conducta; Rendimiento escolar; Adolescencia. 


\section{Introdução}

A adolescência, consolidada como um momento de importantes transformações físicas e psicológicas, especialmente no que diz respeito ao desenvolvimento e ajustamento socioemocional (Pereira, Cia, \& Barham, 2008), costuma ser considerada como um período de risco para o desenvolvimento de problemas emocionais e de comportamento (Wang et al., 2014), os quais podem impactar no rendimento escolar (Marturano \& Elias, 2016). Os problemas emocionais e de comportamento classificam-se em dois grupos, de acordo com a especificidade de seus sintomas, podendo ser do tipo internalizante e externalizante. $\mathrm{O}$ primeiro envolve depressão, ansiedade, retraimento social, queixas somáticas, medo, preocupação em excesso, tristeza, timidez, insegurança e recusa escolar. Já o segundo relaciona-se à oposição, agressão, impulsividade e manifestações antissociais, como provocações e brigas (Achenbach \& Edelbrock, 1979).

Tais sintomas mostram-se associados ao baixo rendimento escolar (Barreto, Freitas, \& Del Prette, 2011; Santos \& Graminha, 2006), visto que os alunos com problemas externalizantes podem interferir na dinâmica da sala de aula (Alvarenga \& Piccinini, 2009; Fuentes, Gázquez, Mercader, Molero, \& Rubira, 2011) e ter a qualidade das relações com seus pares e professores comprometida (Bordin et al., 2013). Além disso, por serem, em geral, agressivos, impulsivos e facilmente distraídos, apresentam dificuldade de permanecerem em sala de aula e se engajarem nas atividades (Dessen \& Szelbracikowski, 2006), podendo ocasionar prejuízos nas funções executivas como memória de trabalho, flexibilidade mental e controle inibitório (FernándezVilar, Carranza-Carnicero, \& Ato-García, 2012). Já os alunos com problemas internalizantes também podem ter seu processo de aprendizagem prejudicado devido a maior dificuldade de fazer perguntas, tirar dúvidas e pedir ajuda aos professores e colegas (Barreto et al., 2011). Consequentemente, podem evitar ir à escola (Moksnes, Lohre, Lillefjell, Byrne, \& Haugan, 2016) e apresentar prejuízos cognitivos, já que os problemas de atenção estão associados com transtorno de ansiedade e depressão (Ribeiro et al., 2017)

Estudos internacionais (Moksnes et al., 2016; Shek \& Li, 2016) indicaram que a cada ano escolar, a amplitude de conhecimento avança e alcançar um bom rendimento torna-se difícil entre os adolescentes devido ao estresse ocasionado pelas exigências acadêmicas e interação com diferentes professores, o que pode se associar a sintomas depressivos e reduzida satisfação com a vida. Nesse sentido, algumas pesquisas se preocuparam em avaliar iniciadores de problemas emocionais e de comportamento e rendimento escolar de forma transversal (Cia \& Costa, 2012; Cruvinel \& Boruchovitch, 2004). Por exemplo, Saleem e Mahmood, (2012) apontaram que os sintomas internalizantes relacionavam-se com a aprendizagem na adolescência, destacando o sentimento de rejeição como uma variável importante para explicar o baixo rendimento escolar. Em contrapartida, estudos longitudinais verificaram a relação do comportamento externalizante com o baixo rendimento escolar em anos subsequentes, devido à dificuldade de se engajarem nas tarefas escolares (Burt \& Roisman, 2010; Malinauskienea, Vosylis, \& Zukauskiene, 2011).

Pesquisas encontradas em âmbito brasileiro, por sua vez, constataram que, de acordo com a avalição dos professores, adolescentes com baixo rendimento escolar apresentavam indicadores de sintomas internalizantes e externalizantes (Barreto et al., 2011). Entretanto, encontram-se estudos que focalizaram apenas um tipo de sintomatologia. Por exemplo, os resultados da pesquisa transversal desenvolvida por Cruvinel e Boruchovitch (2004), que examinou a relação entre sintomas depressivos, estratégias de aprendizagem e rendimento escolar em crianças e adolescentes, evidenciou que os sintomas depressivos são os que mais interferem no repertório de estratégias de aprendizagem e no rendimento em matemática.

$\mathrm{O}$ que parece ser consenso é que os adolescentes com bom rendimento escolar tendem a apresentar menos problemas de comportamento e maior aceitação pelos pares quando comparados a adolescentes com baixo rendimento escolar (Bandeira, Rocha, Souza, Del Prette, \& Del Prette, 2006; Feitosa, Matos, Del Prette, \& Del Prette, 2009). No entanto, não há concordância na literatura sobre o que pode ser considerado causa ou efeito na relação entre problemas emocionais e de comportamento e rendimento escolar, isto é, se os problemas emocionais e de comportamento levam a um pior rendimento escolar (Breslau, Miller, Breslau, Bohnert, Lucia, \& Schweitzer, 2009; Cia \& Costa, 2012) ou se o baixo rendimento escolar propicia o desenvolvimento de tais problemas (Teixeira, Seraceni, Suriano, Sant'ana, Carreiro, \& Paula, 2014). Assim como defenderam algumas pesquisas (Algozzine, Wang, e Violette 2010; Santos \& Graminha, 2006), acredita-se que haja uma inter-relação entre tais variáveis, considerando que uma se apresenta como condição de risco para a outra.

Outra questão relevante diz respeito ao fato de os estudos nacionais sobre os problemas emocionais e de comportamento e rendimento escolar em adolescentes privilegiarem apenas a avaliação de professores (Cia \& Costa, 2012) ou dos próprios alunos e seus professores 
conjuntamente (Barreto et al., 2011; Pereira et al., 2008). As pesquisas encontradas que consideraram a percepção de familiares contemplaram apenas crianças (Bandeira, et al., 2006; Feitosa, Del Prette, Del Prette, \& Loureiro, 2011; Santos \& Graminha, 2006). Desse modo, acredita-se ser importante desenvolver estudos que envolvam diferentes informantes a fim de fornecer um quadro mais detalhado desses problemas em diferentes contextos e nortear intervenções potencialmente efetivas, além de minimizar a influência das diversas expectativas e possíveis vieses dos pesquisadores (Gomez, Vance, \& Gomez, 2014; Souza, Abade, Silva, \& Furtado, 2011). Nessa perspectiva, familiares e professores, por pertencerem a importantes contextos de desenvolvimento, como família e escola, são observadores privilegiados do comportamento dos adolescentes (Major \& SeabraSantos, 2014). Já no que concerne aos adolescentes, a avaliação sobre seus próprios comportamentos pode trazer contribuições relevantes para a compreensão dos indicadores avaliados (Grigorenko, Geiser, Slobodskaya, \& Francis, 2010).

Tendo em vista o exposto, o objetivo deste estudo foi avaliar a relação entre os problemas emocionais e de comportamento e o rendimento escolar de adolescentes que estão cursando os anos finais do ensino fundamental a partir da avaliação destes, seus familiares e professores. A escolha pelos anos finais do ensino fundamental justifica-se pelo desenvolvimento cognitivo, uma vez que nesta etapa os alunos passam a desenvolver habilidades de formular conteúdos puramente abstratos com elevados conceitos matemáticos e filosóficos, diferentemente daqueles matriculados nos anos iniciais que ainda estão em construção do processo de alfabetização e raciocínio lógico, o que poderia interferir na avaliação do rendimento escolar.

\section{Método}

\section{Delineamento e Participantes}

Este estudo apresenta delineamento transversal do tipo correlacional e comparativo, com abordagem quantitativa (Sampieri, Collado, \& Lucio, 2013). Caracteriza-se como correlacional tendo em vista que irá verificar qual a relação entre os problemas emocionais e de comportamento e o rendimento escolar, e como comparativo porque considera diferentes grupos de pais, adolescentes e professores na identificação dos problemas considerados. Compuseram a amostra 161 participantes: 70 adolescentes, 70 famílias, representadas pelos principais cuidadores, e 21 professores, que avaliaram o número correspondente de alunos contemplados nesta pesquisa. Os adolescentes eram provenientes de duas escolas públicas localizadas em um bairro de classe média-baixa da cidade de Porto Alegre e tinham idade entre 11 e 17 anos $(M=12,90$; $\mathrm{DP}=1,08)$, sendo 41 do sexo feminino $(58,6 \%)$ e 29 do sexo masculino (41,4\%). Com relação a sua escolaridade, $18(25,7 \%)$ cursavam o sexto ano e 52 $(74,3 \%)$ o sétimo ano do ensino fundamental. No ano letivo da realização da pesquisa (2015), $13(18,6 \%)$ deles reprovaram e 57 foram aprovados $(81,4 \%)$.

As famílias foram representadas pelo principal cuidador: 61 mães $(87,2 \%)$, sete pais $(10 \%)$, uma tia materna $(1,4 \%)$ e uma avó materna $(1,4 \%)$. Entre os professores, os quais ministravam diferentes disciplinas para as classes participantes, contemplando todas do currículo, cinco $(23,81 \%)$ eram do sexo masculino e $16(76,19 \%)$ do sexo feminino. Todos tinham contato com os alunos avaliados a pelo menos seis meses antes da realização da pesquisa.

A amostra foi acessada por conveniência e estimada de acordo com os critérios propostos por Hair, Black, Babin, Anderson e Thatam (2009) para análise de variância, os quais consideram que o tamanho mínimo exigido é de 20 sujeitos em cada grupo para este tipo de análise. Foram excluídos dois adolescentes com quadro de deficiência mental e transtorno do espectro autista, que apresentavam diagnósticos anterior à pesquisa $\mathrm{e}$ estavam em Atendimento Educacional Especializado - AEE (Brasil, 2008) por necessitarem de estratégias curriculares diferenciadas.

\section{Instrumentos}

Registro do Rendimento Escolar: foram consideradas as notas dos três trimestres das disciplinas de matemática e português referentes ao ano letivo da realização da pesquisa, as quais foram obtidas na secretaria das escolas. A média final para aprovação das escolas participantes era 50 pontos, o que possibilitou classificar o rendimento acadêmico em fraco (0-49 pontos), médio (50-69 pontos) e alto (acima de 70 pontos). Optou-se pelas disciplinas de português e matemática, tendo em vista que a compreensão da leitura contribui para a aprendizagem de diferentes conteúdos escolares e facilita a utilização de processos cognitivos complexos, como, por exemplo, o raciocínio analógico e o analítico (Oliveira, Boruchovitch, \& Santos, 2008). Já a construção do conhecimento matemático requer que o aluno pense sobre possibilidades, estabeleça relações, compreenda os princípios subjacentes às operações e utilize estratégias de resolução de problemas (Corso, 2008).

Inventário de Comportamentos Autorreferidos para Jovens de 11 a 18 Anos (Youth Self Report - 
YSR, Achenbach \& Rescorla, 2001): avalia a saúde mental de adolescentes com idade entre 11 e 18 anos. $\mathrm{O}$ instrumento possui dois níveis de avaliação: 1) competências e 2) indicadores autorreferidos de problemas emocionais e de comportamento a partir de oito escalas que compõem três índices gerais: problemas internalizantes (ansiedade/depressão, isolamento/depressão e queixas somáticas), problemas externalizantes (comportamento desviante e comportamento agressivo) e total de problemas, que engloba todos os itens de problemas de comportamento, além dos problemas sociais, problemas de pensamento e problemas de atenção. Para fins do presente estudo, foi utilizado apenas o segundo nível do inventário. O YSR se encontra em processo de validação para população brasileira, porém apresenta validade da sua estrutura fatorial (RMSEA $=0,03$ ) indicando bom ajustamento (Rocha, 2012). Além disso, Bordin e colaboradores (2013) também revelaram que a confiabilidade média de teste-reteste foi de 0,82 e a consistência interna das escalas de problemas, medido por alfa de Cronbach, variou de 0,67 a 0,95 .

Inventário dos Comportamentos de Crianças e Adolescentes entre Seis e 18 Anos (Child Behavior Checklist - CBCL, Achenbach \& Rescorla, 2001): afere a competência social e a presença de problemas emocionais e de comportamento em crianças e adolescentes de acordo com a percepção dos pais/ cuidadores. Da mesma forma que para o YSR, foi utilizado apenas o segundo nível do inventário. Embora não haja validação do instrumento para a população brasileira, apenas uma análise preliminar (Bordin, Mari, \& Caeiro, 1995), o estudo de Rocha et al. (2013) evidenciou a validade da sua estrutura fatorial $($ RMSEA $=0,02)$ e capacidade discriminativa $(p<001)$. Silvares, Rocha e Emerich (2016) também revelaram que foram encontrados bons índices de consistência interna de 0,95 para amostras não encaminhadas para serviços de saúde mental e 0,95 para uma amostra de crianças e adolescentes encaminhada para atendimento em um serviço de psicologia de Porto Alegre/RS.

Inventário dos Comportamentos de Crianças e Adolescentes - Relatório para Professores (Teacher's Report Form - TRF, Achenbach \& Rescorla, 2001): avalia o funcionamento adaptativo, desempenho acadêmico, problemas internalizantes e problemas externalizantes. $\mathrm{O}$ instrumento se constitui como uma medida paralela ao CBCL, que foi desenvolvida para, quando usados em conjunto, proporcionar uma compreensão global do funcionamento da criança/ adolescente em diferentes ambientes, devendo ser respondido pelo professor que conheça o aluno há pelo menos dois meses, critério que foi cumprido no presente estudo. Bordin et al. (2013) afirmam que o instrumento é considerado padrão ouro para a avaliação de problemas de comportamento e é um dos mais utilizados no mundo, com boa confiabilidade teste-reteste $(0,90) \mathrm{e}$ consistência interna, medida pelo alfa de Cronbach, variando de 0,72 a 0,97. Entretanto, as validações do instrumento estão sendo conduzidas no Brasil por pesquisadores das Universidades Estadual e Federal de São Paulo (USP e Unifesp), sendo comparados os itens das versões traduzidas pelos grupos de pesquisadores para posterior análise e finalização da versão brasileira.

\section{Procedimentos de Coleta de Dados}

Inicialmente, foi realizado contato presencial com a Secretaria de Educação do Estado do Rio Grande do Sul, com vistas à autorização do estudo e duas escolas da cidade de Porto Alegre/RS foram selecionadas por conveniência, considerando a localização acessível à pesquisadora. As famílias foram contatadas por meio de palestras informativas, além de reuniões para entrega de avaliações. Aqueles que aceitavam participar assinavam o Termo de Consentimento Livre e Esclarecido (TCLE) e levavam um envelope constando uma Carta de Apresentação da Pesquisa, instruções quanto aos procedimentos de preenchimento do CBCL e o instrumento propriamente dito. O prazo de preenchimento para os responsáveis foi de uma semana e, depois, deveriam reenviar pelos adolescentes. O material era recolhido na própria escola. Os adolescentes, por sua vez, foram acessados em sala de aula, com a devida liberação da Direção da escola e aprovação do professor responsável pela disciplina, para assinarem o Termo de Assentimento e responderem coletivamente ao YSR. Por fim, com os professores foi agendado um dia e horário que estivessem disponíveis na escola para assinarem o Termo de Consentimento Livre e Esclarecido e responderem ao TRF. Tendo em vista a extensão do instrumento, o período do início da coleta (terceiro trimestre), e considerando que muitos dos adolescentes participantes faziam parte de uma mesma turma, todos os professores dos anos finais foram orientados individualmente sobre quem deveriam considerar para avaliação a fim de não sobrecarregá-los. Assim, nenhum deles preencheu a mais de sete inventários. Destaca-se que o presente estudo foi avaliado e aprovado pelo Comitê de Ética em Pesquisa da Universidade do Vale do Rio dos Sinos, sob o CAAE 47369215.2.0000.5344.

\section{Procedimentos de Análise dos Dados}

As respostas dos instrumentos YSR, CBCL e TRF foram analisadas a partir do ASEBA-PC. Para 
fins deste estudo, não foram consideradas as escalas orientadas pelos critérios do Manual Diagnóstico e Estatístico de Transtornos Mentais (DSM-IV). Os escores das escalas contempladas podem ser classificados em clínico (percentis a partir de 70), borderline ou limítrofe (percentis entre 65 a 69) e não clínico (percentis abaixo de 65), mas neste estudo optou por incluir os adolescentes categorizados como limítrofes na categoria clínica, conforme recomendação de Achenbach e Rescorla (2001). Essa classificação não representa um diagnóstico, porém auxilia na identificação de fatores de risco para se considerar a necessidade de ajuda profissional. Além disso, foram utilizados na base de dados da pesquisa os escores $T$ referentes a cada escala dos instrumentos, visto que esse padroniza os resultados, não havendo interferência do número de itens das escalas (Rocha, 2012).

Para estatística descritiva foram utilizadas as distribuições absoluta (n) e relativa (\%), bem como as medidas de tendência central e dispersão (média, mediana e desvio padrão) com o estudo de normalidade da distribuição dos dados pelo teste de KolmorogovSmirnov (correção de Lillifors). Com o objetivo de verificar a existência de relação entre as escalas do YSR, CBCL e TRF e a média final obtida nas disciplinas de português e matemática, realizou-se a análise de correlação de Pearson. Após, foi utilizado o teste Qui-quadrado de Pearson ou Exato de Fisher para comparar o rendimento acadêmico (fraco, médio, alto), considerando a análise de forma independente (português e matemática separadamente) e conjunta (média final de ambas as disciplinas), com as escalas do YSR, CBCL e TRF. Por fim, os adolescentes foram classificados em dois grupos, aprovados e reprovados e utilizou-se o teste $t$ de Student para amostras independentes.

\section{Resultados}

As relações entre as escalas do YSR, CBCL, TRF e a nota final obtida nas disciplinas de português e matemática, foram realizadas de acordo com a análise de correlação de Pearson. Como pode se verificar na Tabela 1, foram encontradas correlações significativas e negativas, para a escala problemas de atenção na avaliação de adolescentes $(p<0.01)$, professores $(p<0.01)$ e familiares $(p<0.01)$. Os docentes também evidenciaram correlações negativas para comportamento desviante $(p<0.01)$, comportamento agressivo $(p<0.04)$ e problemas externalizantes $(p<0,01)$. Para o total de problemas evidenciou-se correlação negativa na avaliação de adolescentes $(p<0.02)$ e professores $(p<0.01)$. Sobre as demais escalas dos instrumentos, nas três esferas avaliadas, as relações com a nota final não se mostraram significativas.

\section{TABELA 1}

Correlação de Pearson entre Nota Final e Avaliação dos Problemas Emocionais e de Comportamento

\begin{tabular}{lccc}
\hline \multirow{2}{*}{ Escalas } & \multicolumn{3}{c}{ Coeficiente de correlação $(r)$} \\
\cline { 2 - 4 } & Adolescentes & Familiares & Professores \\
Ansiedade/depressão & -0.15 & -0.06 & -0.03 \\
Isolamento/depressão & -0.10 & -0.16 & -0.16 \\
Queixas somáticas & -0.15 & -0.04 & 0.10 \\
Problemas sociais & -0.18 & -0.11 & -0.20 \\
Problemas de pensamento & -0.18 & -0.03 & -0.12 \\
Problema de atenção & $-0.34^{*}$ & $-0.37^{*}$ & $-0.52^{*}$ \\
Comportamento desviante & -0.22 & -0.14 & $-0.31^{*}$ \\
Comportamento agressivo & -0.10 & -0.14 & $-0.24^{*}$ \\
Problemas internalizantes & -0.15 & -0.01 & -0.05 \\
Problemas externalizantes & -0.21 & -0.19 & $-0.29^{*}$ \\
Total de problemas & $-0.26^{*}$ & -0.17 & $-0.33^{*}$ \\
\hline
\end{tabular}

${ }^{*} p<0.05$.

Quanto à comparação do rendimento acadêmico (fraco, médio e alto) nas disciplinas de português e matemática (avaliadas em conjunto), com as respostas dos adolescentes, familiares e professores, por meio do teste Qui-quadrado de Pearson, foi observada diferença significativa na avaliação dos professores, apontando que os alunos com rendimento fraco foram identificados com a classificação clínica para problemas de atenção $\left[\chi^{2}(4, n=4)=11,066, p=0.03\right]$. $\mathrm{Na}$ avaliação de familiares e adolescentes, não foram encontradas diferenças estatísticas significativas.

Os escores dos problemas emocionais e de comportamento avaliados por adolescentes, familiares e professores também foram comparados com a classificação das notas nas disciplinas de português e matemática separadamente. Os resultados não evidenciaram diferença significativa na avaliação dos adolescentes, familiares e professores quanto a classificação da nota de português e as escalas dos instrumentos. Com relação ao rendimento em matemática, foi observada diferença na escala problemas de atenção a partir da avaliação dos adolescentes, indicando que os alunos com nota abaixo da média $(n=3)$ ou média $(n=13)$ classificavam-se como clínicos para problemas de atenção (Teste Exato de Fisher; $p<0.03$ ). Além disso, os professores indicaram que os adolescentes com rendimento fraco e médio em matemática mostraram-se como clínicos para problemas de atenção $\left[\chi^{2}(4, \mathrm{n}=9)=13,500, p<0.01\right]$, comportamento des- 
viante $\left[\chi^{2}(4, \mathrm{n}=6)=15,483, p<0.01\right]$ e problemas externalizantes $\left[\chi^{2}(4, \mathrm{n}=13)=11,695, p<0.02\right]$. Já na avaliação dos familiares, foi evidenciado que os adolescentes com rendimento fraco $(n=5)$ e médio $(n=17)$ na disciplina mostraram-se como clínicos somente para problemas externalizantes (Teste Exato de Fisher, $p<0.02$ ).

Por fim, buscou-se comparar as respostas de adolescentes, familiares e professores sobre os problemas emocionais e de comportamento com o grupo de alunos reprovados no ano letivo $(\mathrm{n}=13)$ e aprovados $(\mathrm{n}=57)$ por meio do teste $t$ de Student para amostras independentes. Verificou-se que na avaliação dos adolescentes, tanto o grupo de reprovados quanto de aprovados não se mostraram diferentes quanto aos problemas emocionais e de comportamento. Já na avaliação dos familiares, os resultados evidenciaram diferença entre os grupos, indicando que os alunos com reprovação tinham mais indicadores de problemas de atenção. No que se refere a avaliação dos professores, revelou-se que no grupo de alunos reprovados havia mais indicadores de problemas de atenção, comportamento desviante, problemas externalizantes e total de problemas. Na Tabela 2 constam os resultados que foram significativos.

\section{Discussão}

Como sabido, o presente estudo teve por objetivo avaliar a relação entre os problemas emocionais, de comportamento e rendimento escolar de adolescentes do ensino fundamental a partir da avaliação destes, seus familiares e professores. Em conjunto, os resultados encontrados corroboram a literatura da área, especialmente no que diz respeito à associação entre problemas de atenção (Barreto et al., 2011; Santos \& Graminha, 2006) e sintomas externalizantes (Cia \& Costa, 2012; Dazzani, Cunha, Luttigards, Zucoloto, \& Santos, 2014; Malinauskienea et al., 2011) e o baixo rendimento escolar de adolescentes.

Destaca-se que os indicadores de problemas de atenção foram os únicos que os três informantes relacionaram negativamente ao rendimento escolar, em grau fraco para os adolescentes e os familiares, e moderado para os professores. Breslau et al., (2009) já haviam evidenciado que os problemas de atenção podem influenciar negativamente $o$ rendimento escolar na adolescência, gerando reduzidas expectativas com relação ao sucesso escolar tanto para o adolescente quanto para seus familiares e professores. Consequentemente, os estudantes podem

TABELA 2

Teste $t$ de Student para amostras independentes segundo a variável reprovado e aprovado

\begin{tabular}{|c|c|c|c|c|c|c|}
\hline \multirow{2}{*}{ Escalas } & \multicolumn{2}{|c|}{ Reprovado $(n=13)$} & \multicolumn{2}{|c|}{ Aprovado $(n=57)$} & \multirow{2}{*}{$t$} & \multirow{2}{*}{$p$} \\
\hline & Média & $D P$ & Média & $D P$ & & \\
\hline \multicolumn{7}{|c|}{ Problema de atenção } \\
\hline \multicolumn{7}{|l|}{ Adolescentes } \\
\hline Familiares & 61,5 & 5,1 & 57,9 & 7,4 & 2,35 & 0,02 \\
\hline Professores & 62,7 & 8,4 & 55,3 & 5,8 & 3,03 & 0,00 \\
\hline \multicolumn{7}{|c|}{ Comportamento desviante } \\
\hline \multicolumn{7}{|c|}{ Adolescentes } \\
\hline \multicolumn{7}{|l|}{ Familiares } \\
\hline Professores & 59,2 & 7,1 & 54,0 & 5,6 & 2,47 & 0,01 \\
\hline \multicolumn{7}{|c|}{ Problemas externalizantes } \\
\hline \multicolumn{7}{|l|}{ Adolescentes } \\
\hline \multicolumn{7}{|l|}{ Familiares } \\
\hline Professores & 59,4 & 8,0 & 53,2 & 8,3 & 2,46 & 0,01 \\
\hline \multicolumn{7}{|c|}{ Total de problemas } \\
\hline \multicolumn{7}{|l|}{ Adolescentes } \\
\hline \multicolumn{7}{|l|}{ Familiares } \\
\hline Professores & 60,5 & 6,7 & 54,0 & 8,6 & 2,45 & 0,01 \\
\hline
\end{tabular}


perder a motivação para a aprendizagem e tornar-se mais suscetíveis ao interesse por uso de substâncias químicas e abandono escolar (Malinauskiene et al., 2011; Saleem \& Mahmood, 2012), entre outras situações de vulnerabilidade social.

Constatou-se também que o baixo rendimento escolar foi relacionado negativamente com os comportamentos agressivo, desviante e problemas externalizantes pelos professores. Tais resultados corroboraram achados da literatura, que evidenciam que os alunos com problemas externalizantes apresentam dificuldades para lidar com as demandas da escola, pois, devido a característica de seus sintomas, não conseguem permanecer na sala de aula por muito tempo ou se engajar em atividades escolares, o que compromete o seu rendimento escolar (Alvarenga \& Piccinini, 2009; Dessen \& Szelbracikowski, 2006). Além disso, esses alunos tendem a sobrecarregar o professor, especialmente dentro do espaço de sala de aula (Sutherland, Lewis-Palmer, Stichter, \& Morgan, 2008), por apresentarem comportamentos que quebram a rotina de ensino, justificando que sejam julgados por eles como alunos com mais dificuldades. Ainda, eles podem incitar sentimentos de impotência nos docentes, que acabam buscando alternativas como o encaminhamento para avaliação médica ou psicológica, a qual é associada, muitas vezes, à medicalização (Leonardo \& Suzuki, 2016).

De forma semelhante, os familiares associaram o baixo rendimento escolar dos adolescentes aos indicadores de problemas de atenção e problemas externalizantes. É plausível supor que tais dados sejam reveladores do menor contato e interação das famílias com os filhos e seus professores nos anos finais do ensino fundamental, reforçando a suposição de que o adolescente precisa prestar atenção no conteúdo apresentado pelo docente e não perturbar a aula para que consiga garantir um rendimento escolar satisfatório.

Nessa perspectiva, os professores também acabam responsabilizando o aluno pelo baixo rendimento escolar, apontando que a falta de atenção ocorre por vontade própria (Leonardo \& Suzuki, 2016). Consequentemente, os próprios adolescentes tendem a se responsabilizar pelo seu desempenho, atribuindo suas dificuldades a fatores internos (incapacidade para aprender e possibilidade de ter um problema de origem orgânica) e externos (deixar de realizar os deveres escolares), o que pode justificar a associação do baixo rendimento escolar com os problemas de atenção e total de problemas (Osti \& Brenelli, 2013). Contudo, acredita-se que responsabilizar os adolescentes acaba por reforçar os sintomas externalizantes que, quando comparados aos internalizantes, apresentam maior prevalência e estabilidade ao longo do tempo, assim como maior probabilidade de evoluírem para quadros clínicos mais graves na vida adulta (Alvarenga \& Piccinini, 2009). Além disso, a culpabilização do adolescente exclui a possibilidade de reflexão sobre as práticas escolares, impedindo uma compreensão contextualizada do rendimento escolar (Dazzani et al., 2014).

Outro aspecto importante é o fato de os alunos reprovados da amostra terem sido avaliados por seus familiares e professores como tendo mais indicadores de problemas de atenção, do mesmo modo que estes últimos apontaram indicadores de comportamento desviante, problemas externalizantes e total de problemas. Para Santos e Sant'Ana (2013) a reprovação escolar está associada a sentimentos de descrença em relação a capacidade de aprender e pode estar relacionada a vivência de fracasso (Pezzi, Donelli, \& Marin, 2016).

No que diz respeito aos resultados da comparação entre as disciplinas de matemática e português com os problemas emocionais e de comportamento da amostra, encontrou-se que os professores e os adolescentes indicaram problemas de atenção, bem como os familiares e os docentes evidenciaram problemas externalizantes para alunos com nota abaixo da média ou na média em matemática. Pereira et al. (2008) contribuem para o entendimento de tais achados ao sugerirem que o aprendizado da matemática requer habilidades de comunicação (escutar, questionar, compreender novos conceitos), prestar atenção e mostrar persistência, indicando a relação positiva entre estas habilidades e rendimento escolar. Por outro lado, quanto a nota de português, os resultados não evidenciaram diferença significativa na avaliação dos adolescentes, familiares e professores no que concerne aos problemas emocionais e de comportamento. Esse dado vai ao encontro do estudo longitudinal de Algozzine et al. (2010) em que não foi confirmada a influência dos problemas de comportamento sobre o desempenho da leitura de um ano escolar para outro, destacando a concepção que o rendimento escolar e os problemas de comportamento podem ser ambientalmente mediados.

Como visto, evidencia-se a inter-relação entre os problemas emocionais e de comportamento e o rendimento escolar, uma vez que tais problemas acabam por interferir no rendimento escolar dos alunos, assim como a demanda acadêmica e o baixo rendimento escolar podem acentuá-los (Santos \& Graminha, 2006). Outro aspecto a ser considerado é a pouca ênfase nos resultados em relação aos sintomas internalizantes. Apesar de não serem encontradas diferenças significativas na avaliação dos adolescentes, 
pais e professores quanto a associação entre os sintomas internalizantes e o rendimento escolar, tais comportamentos foram identificados. De acordo com Borba e Marin (2018), os adolescentes evidenciam mais indicadores de problemas internalizantes que seus familiares e professores, possivelmente por serem mais restritos ao mundo interno dos adolescentes (AlckminCarvalho et al., 2014), assim como subestimados em relação a sua severidade no contexto escolar, uma vez que chamam menos atenção em sala de aula (Lyra, Assis, Njaine, \& Pires, 2013). De qualquer forma, a presente pesquisa revelou que os indicadores externalizantes e os problemas de atenção continuam sendo destacados quando relacionados às questões acadêmicas.

Torna-se importante apontar algumas limitações do estudo, visto que não é possível afirmar se a dificuldade escolar dos alunos participantes pode ser entendida como uma consequência das dificuldades nos processos de aprendizagem durante os anos iniciais do ensino fundamental, já que não foi realizada uma avaliação cognitiva dos adolescentes, ou se é desencadeada pelas adversidades escolares do contexto público considerado, como greves, falta de professores e de recursos didáticos (Moscaritolo, Rocha, \& Silvares, 2013). Cia e Costa (2012) pontuaram alguns aspectos como pouca ênfase do professor em trabalhos escolares, recursos didáticos pobres, greve escolar e mudança de professor como fatores de risco à ocorrência ou manutenção de problemas de comportamento dos alunos e sugere-se que novos estudos possam contemplar tais questões.

Por fim, destaca-se que o presente estudo oferece subsídios que contribuem para uma melhor compreensão sobre os problemas emocionais, de comportamento e o rendimento escolar de adolescentes, uma vez que parte da perspectiva de múltiplos informantes. Dessa maneira, responsabilizar somente o adolescente, sua família ou a escola pelos problemas emocionais e de comportamento e o baixo rendimento escolar representa uma barreira na compreensão do fenômeno, o que dificulta a reformulação de práticas pedagógicas que envolvam todos os sistemas envolvidos na promoção da saúde mental dos estudantes. Acreditase que os resultados apresentados podem fundamentar ações de prevenção em saúde mental nas escolas, já que indicaram condições de risco emocional, comportamental e escolar entre adolescentes.

\section{Referências}

Achenbach, T. M. \& Edelbrock, C. S. (1979). The child behavior profile: II. Boys aged 12-16 and girls aged 6-11 and 12-16. Journal of Consulting and Clinical Psychology, 47(2), 223-233. https://doi.org/10.1037/0022-006X.47. 2.223

Achenbach, T. M. \& Rescorla, L. A. (2001). Manual for the ASEBA school-age forms, \& profiles. Burlington, VT: University of Vermont, Research Center for Children, Youth, \& Families.

Alckmin- Carvalho, F., Izbicki, S., \& Melo, M. H. S. (2014). Problemas de comportamento segundo vítimas de bullying e seus professores. Estudos e Pesquisas em Psicologia, 14(3), 834-853. Retrieved from http://www.e-publicacoes. uerj.br/index.php/revispsi/article/view/13886/10607. https://doi.org/10.12957/epp.2014.13886

Algozzine, B., Wang, C., \& Violette, A. S. (2010). Reexamining the relationship between academic achievement and social behavior. Journal of Positive Behavior Interventions, 13, 1-14. https://doi.org/10.1177/1098300709359084

Alvarenga, P. \& Piccinini, C. A. (2009). Práticas educativas maternas e indicadores do desenvolvimento social no terceiro ano de vida. Psicologia: Reflexão e Crítica, 22(2), 191-199. https://doi.org/10.1590/S0102-79722009000200004

Bandeira, M., Rocha, S. S., Souza, T. M., Del Prette, Z. A., \& Del Prette, A. (2006). Comportamentos problemáticos em estudantes do ensino fundamental: Características da ocorrência e relação com habilidades sociais e dificuldades de aprendizagem. Estudos de Psicologia, 11(2), 199-208. https://doi.org/10.1590/S1413-294X2006000200009

Barreto, S. O., Freitas, L. C., \& Del Prette, Z. A. (2011). Habilidades sociais na comorbidade entre dificuldades de aprendizagem e problemas de comportamento: Uma avaliação multimodal. Psico, 42(4), 503-510.

Borba, B. M. \& Marin, A. H. (2018). Indicadores de problemas emocionais e de comportamento em adolescentes: Concordância entre múltiplos informantes. Paidéia, 28(e2825). https://dx.doi.org/10.1590/1982-4327e2825

Bordin, I. A. S, Mari, J. J., \& Caeiro, M. F. (1995). Validação da versão brasileira do Child Behavior Checklist (CBCL) (Inventário de Comportamentos da Infância e Adolescência): Dados preliminares. Revista da Associação Brasileira de Psiquiatria - Asociación Psiquiatrica de la America Latina, 17(2), 55-66.

Bordin, I. A., Rocha, M. M., Paula, C. S., Teixeira, M. C. T. V., Achenbach, T. M., Rescorla, L. A., \& Silvares, E. F. M. (2013). Child behavior checklist (CBCL), youth self-report (YSR) and teacher's report form (TRF): An overview of the development of the original and Brazilian versions. Cadernos de Saúde Pública, 29(1), 13-28. https://doi. org/10.1590/S0102-311X2013000100004

Brasil. (2008). Política nacional de educação especial na perspectiva da educação inclusiva. Brasília: MEC/SEESP. 
Breslau, J., Miller, E., Breslau, N., Bohnert, K., Lucia, V., \& Schweitzer, J. (2009). The impact of early behavior disturbances on academic achievement in high school. Pediatrics, 123(6), 1472-1476. https://doi.org/10.1542/ peds.2008-1406

Burt, K. B. \& Roisman, G. I. (2010). Competence and psychopathology: Cascade effects in the NICHD Study of early childcare and youth development. Development and Psychopathology, 22, 557-567. https://doi.org/10.1017/ S0954579410000271

Cia, F. \& Costa, C. S. L. (2012). Desempenho acadêmico nas séries do ensino fundamental: Relação com o desenvolvimento social. Psicologia Argumento, 30(68), 109-120. https://doi.org/10.7213/psicol.argum.5889

Corso L. V. (2008). Dificuldades na leitura e na matemática: Um estudo dos processos cognitivos em alunos da $3 \underline{a}$ a $6^{a}$ série do Ensino Fundamental. Tese [Doutorado], Universidade Federal do Rio Grande do Sul, Porto Alegre, RS, Brasil.

Cruvinel, M. \& Boruchovitch, E. C. (2004). Sintomas depressivos, estratégias de aprendizagem e rendimento escolar de alunos do ensino fundamental. Psicologia em Estudo, 9, 369-378. https://doi.org/10.1590/S1413-73722004000300005

Dazzani, M. V. M., Cunha, E. O., Luttigards, P. M., Zucoloto, P. C. S. V., \& Santos, G. L. (2014). Queixa escolar: Uma revisão crítica da produção científica nacional. Revista Quadrimestral da Associação Brasileira de Psicologia Escolar e Educacional, 18(3), 421-428. https://doi.org/10.1590/2175-3539/2014/0183762

Dessen, M. A. \& Szelbracikowski, A. C. (2006). Estabilidades e mudanças em padrões familiares de crianças com problemas de comportamento exteriorizado. Paidéia, 16(33), 71-80. https://doi.org/10.1590/S0103-863X20060001 00010

Feitosa, F. B., Del Prette, Z. A. P., Del Prette, A., \& Loureiro, S. R (2011). Explorando relações entre comportamento social e o desempenho acadêmico em crianças. Estudos e Pesquisas em Psicologia, 11(2), 442-455. https://doi. org/10.12957/epp.2011.8383

Feitosa, F. B., Matos, M. G., Del Prette, Z. A. P., \& Del Prette, A. (2009). Desempenho acadêmico e interpessoal em adolescentes portugueses. Psicologia em Estudo, 14(2), 259-266. https://doi.org/10.1590/S1413-73722009000200006

Fernández-Vilar, M. \& Carranza-Carnicero, J., \& Ato-García, M. (2012). Efecto del ajuste socioemocional en el rendimiento y las competencias académicas en el contexto escolar: Estudio comparativo. Anales de Psicología, 28(3), 892-903. https://doi.org/10.6018/analesps.28.3.131211

Fuentes, M. C. P., Gázquez, J. J., Mercader, I., Molero, M. M., \& Rubira, M. M. G. (2011). Rendimiento académico y conductas antisociales y delictivas en alumnos de educación secundaria obligatoria. International Journal of Psychology and Psychological Therapy, 11(3), 401-412.

Frizzo, G. B., Pedrini, J. R., Souza, D. S., Bandeira, D. R., \& Borsa, J. C. (2014). Reliability of child behavior checklist and teacher's report form in a sample of Brazilian children. Universitas Psychologica, 14(1), 149-156. https://doi. org/10.11144/Javeriana.upsy14-1.rcbc

Gomez, R., Vance, A., \& Gomez, R. M. (2014). Analysis of the convergent and discriminant validity of the CBCL, TRF, and YSR in a clinic-referred sample. Journal of Abnormal Child Psychology, 42(8), 1413-1425. https://doi. org/10.1007/s10802-014-9879-4

Grigorenko, E. L., Geiser, C., Slobodskaya, H. R., \& Francis, D. J. (2010). Cross-informant symptoms from CBCL, TRF, and YSR: Trait and method variance in a normative sample of Russian youths. Psychological Assessment, 22(4), 893-911. https://doi.org/10.1037/a0020703

Hair, J. F., Black, W. C., Babin, B. J., Anderson, R. E., \& Thatam, R. L. (2009). Análise Multivariada de Dados. Porto Alegre: Bookman.

Leonardo, N. S. T. \& Suzuki, M. A. (2016). Medicalização dos problemas de comportamento na escola: Perspectivas de professores. Fractal: Revista de Psicologia, 28(1), 46-54. https://doi.org/10.1590/1984-0292/1161

Lyra, G. F. D., Assis, S. G., Njaine, K., \& Pires, T. O. (2013). Sofrimento psíquico e trabalho docente: Implicações na detecção de problemas de comportamento em alunos. Estudos e Pesquisas em Psicologia, 13(2), 724-744. https:// doi.org/10.12957/epp.2013.8433

Major, S. \& Seabra-Santos, M. J. (2014). Pais e/ou professores? Acordo entre informadores na avaliação socioemocional de pré-escolares [Parents and/or teachers? Inter-ratter agreement on preschoolers social-emotional assessment]. Psicologia: Teoria e Pesquisa, 30(4), 373-383. https://doi.org/10.1590/S0102-37722014000400002

Malinauskienea, O., Vosylisa, R., \& Zukauskienea, R. (2011). Longitudinal examination of relationships between problem behaviors and academic achievement in young adolescents. Procedia Social and Behavioral Sciences, 15, 415-342. https://doi.org/10.1016/j.sbspro.2011.04.311

Marturano, E. M. \& Elias, L. C. S. (2016). Família, dificuldades no aprendizado e problemas de comportamento em escolares. Educar em Revista, 59, 123-139. https://doi.org/10.1590/0104-4060.44617

Moksnes, U. K., Lohre, A., Lillefjell, M, Byrne, D. G., \& Haugan, G. (2016). The association between school stress, life satisfaction and depressive symptoms in adolescents: Life satisfaction as a potential mediator. Social Indicators Research, 125(1), 339-357. https://doi.org/10.1007/s11205-014-0842-0

Moscaritolo, A. M. F., Rocha, M. M., \& Silvares, E. F. M. (2013). Indicadores de autoconceito em adolescentes: Autorrelato sobre aspectos positivos e preocupações. Revista Psicologia: Teoria e Prática, 15(3), 134-150.

Oliveira, K. L., Boruchovitch, E., \& Santos, A. A. A. (2008). Leitura e desempenho escolar em português e matemática no ensino fundamental. Paidéia, 18(41), 531-540. https://doi.org/10.1590/S0103-863X2008000300009 
Osti, A. \& Brenell, R. P. (2013). Sentimentos de quem fracassa na escola: Análise das representações de alunos com dificuldades de aprendizagem. Psico-USF, 18(3), 417-426. https://doi.org/10.1590/S1413-82712013000300008

Pereira, C. S. P., Cia, F. \& Elizabeth Joan Barham, E. J. (2008). Autoconceito, habilidades sociais, problemas de comportamento e desempenho acadêmico na puberdade: Inter-relações e diferenças entre sexos. Interação em Psicologia, 12(2), 203-213. https://doi.org/10.5380/psi.v12i2.7870

Pezzi, F. A. S., Marin, A. H., \& Donelli, T. M. S. (2016). School Failure in the Perception of Adolescents, Parents and Teachers. Psico-USF, 21(2), 319-330. https://doi.org/10.1590/1413-82712016210209

Ribeiro, A., Marino, R., Cantiere, C., Teixeira, M., Rocha, M., Schwartzman, J., \& Carreiro, L. (2017). Contribuição de múltiplos informantes para avaliação comportamental de adolescentes com queixas de desatenção e hiperatividade. Psico, 48(4), 295-305. https://doi.org/10.15448/1980-8623.2017.4.25859

Rocha, M. M. (2012). Evidências de validade do "Inventário de autoavaliação para adolescentes" (YSR/2001) para a população brasileira. Tese [Doutorado], Instituto de Psicologia da Universidade de São Paulo, SP, Brasil.

Rocha, M. M., Rescorla, L. A., Emerich, D. R., Silvares, E. F. M., Borsa, J. C., Araújo, L. G., Bertolla M. H., Oliveira M. S., Perez N. C., Freitas P. M., \& Assis, S. G. (2013). Behavioural/emotional problems in Brazilian children: Findings from parents' reports on the Child Behavior Checklist. Epidemiology and Psychiatric Sciences, 22(4), 329-338. https:// doi.org/10.1017/S2045796012000637

Saleem, S. \& Mahmood, Z. (2012). Relationship between emotional and behavioral problems and school performance of adolescents. FWU journal of Social Sciences, 6(2), 187-193.

Sampieri, R. H., Collado, C. F., \& Lucio. M. P. B. (2013). Metodologia de pesquisa. Porto Alegre: Penso.

Santos, P. \& Graminha, S. (2006). Problemas emocionais e comportamentais associados ao baixo rendimento acadêmico. Estudos de Psicologia, 11(1), 101-109. https://doi.org/10.1590/S1413-294X2006000100012

Santos, J. A. \& Sant'Ana, R. B. (2013). Significações da reprovação escolar por alunos adolescentes de escola pública. Revista Educação, 38(3), 691-702. https://doi.org/10.5902/198464445113

Shek, D. T. L. \& Li, X. (2016). Perceived school performance, life satisfaction, and hopelessness: A 4-year longitudinal study of adolescents in hongkong. Social Indicators Research, 126(2), 921-934. https://doi.org/10.1007/s11205-0150904-y

Silvares, E. F. de M., Rocha, M. M. da R., \& Emerich, D. R. (2016). Inventário de comportamento da infância e da adolescência (CBCL), relatório para professores (TRF), inventário de autoavaliação para adolescentes (YSR). In C. Gosrenstein, Y. P. Wang, \& I. Hungerbühler. Instrumentos de avaliação em saúde mental. Porto Alegre: Artmed.

Souza, J., Abade, F., Silva, P. M. C., \& Furtado, E. F. (2011). Avaliação do funcionamento familiar no contexto da saúde mental [Family functioning assessment in the context of mental health]. Revista de Psiquiatria Clinica, 38(6), 254-259. https://doi.org/10.1590/S0101-60832011000600007

Sutherland, K. S., Lewis-Palmer, T., Stichter, J., \& Morgan, P. L. (2008). Examining the influence of teacher behavior and classroom context on the behavioral and academic outcomes for students with emotional or behavioral disorders. The Journal of Special Education, 41(4), 223-233. d https://doi.org/10.1177/0022466907310372

Teixeira, M. C. T. V., Seraceni, M. F. F., Suriano, R., Sant'ana, N. Z., Carreiro, L. R. R., \& Paula, C. S. (2014). Fatores de proteção associados a problemas emocionais e comportamentais em escolares. Estudos de Psicologia, 31(4) 539-548. https://doi.org/10.1590/0103-166X2014000400008

Wang, J., Liu, L., Wu, H., Yang, X., Wang, Y., Wang, L. (2014). Agreement between parents and adolescents on emotional and behavioral problems and its associated factors among Chinese school adolescents: a cross-sectional study. BioMed Central Psychiatry, 14, 114-128. https://doi.org/10.1186/1471-244X-14-114

Dados dos autores:

Bruna Mainardi Rosso Borba - Mestre, Universidade do Vale do Rio dos Sinos.

Angela Helena Marin - Doutora, Universidade do Vale do Rio dos Sinos.

Endereço para correspondência:

Bruna Mainardi Rosso Borba

Av. Unisinos, 950, Sala E01-109 - Bairro Cristo Rei

93022-750 São Leopoldo, RS, Brasil

<brunaborba@yahoo.com.br>

Recebido em: 22.03.2017

Aceito em: 02.04.2018 